\title{
Randomised Clinical Trial: Gut Microbiome Biomarkers are Associated with Clinical Response to a Low FODMAP Diet in Children with Irritable Bowel Syndrome
}

\author{
Bruno P. Chumpitazi, Julia L. Cope, Emily B. Hollister, Cynthia M. Tsai, Ann R. McMeans, \\ Ruth A. Luna, James Versalovic, and Robert J. Shulman \\ Department of Pediatrics, Baylor College of Medicine, Houston, TX, USA (BPC, CMT, RJS); \\ Children's Nutrition Research Center, Houston, TX, USA (ARM, RJS); Department of Pathology, \\ Baylor College of Medicine, Houston, TX, USA (JLC, EBH, RAL, JV); and Texas Children's \\ Microbiome Center, Texas Children's Hospital, Houston, TX, USA (JLC, EBH, RAL, JV)
}

\section{SUMMARY}

Background-A low fermentable oligosaccharides, disaccharides, monosaccharides, and polyols (FODMAP) diet can ameliorate symptoms in adult irritable bowel syndrome (IBS) within 48 hours.

\begin{abstract}
Aim-To determine the efficacy of a low FODMAP diet in childhood IBS and whether gut microbial composition and/or metabolic capacity are associated with its efficacy.

Methods-In a double-blind, crossover trial, children with Rome III IBS completed a one-week baseline period. They then were randomized to a low FODMAP diet or typical American childhood diet (TACD), followed by a 5-day washout period before crossing over to the other diet. GI symptoms were assessed with abdominal pain frequency being the primary outcome. Baseline gut microbial composition (16S rRNA sequencing) and metabolic capacity (PICRUSt) were determined. Metagenomic biomarker discovery (LEfSe) compared Responders ( $250 \%$ decrease in abdominal pain frequency on low FODMAP diet only) versus Non-Responders (no improvement during either intervention).
\end{abstract}

Results-Thirty-three children completed the study. Less abdominal pain occurred during the low FODMAP diet versus TACD $(1.1 \pm 0.2$ (SEM) episodes/day versus $1.7 \pm 0.4, \mathrm{P}<0.05)$. Compared to baseline $(1.4 \pm 0.2)$, children had fewer daily abdominal pain episodes during the low FODMAP diet $(\mathrm{P}<0.01)$ but more episodes during the TACD $(\mathrm{P}<0.01)$. Responders were enriched at baseline in taxa with known greater saccharolytic metabolic capacity (e.g.,

Corresponding Author: Bruno P. Chumpitazi, Assistant Professor of Pediatrics, 6701 Fannin Street, CCC 1010.03, Houston, TX, 77030, chumpita@bcm.edu; phone: 1-832-822-4703.

ClinicalTrials.gov identifier: NCT01339117.

\section{AUTHORSHIP STATEMENT}

Guarantor of the article: Bruno Chumpitazi

Author contributions: BPC and RJS designed the project; BPC, CMT, ARM conducted the research; BPC, JLC, EBH, ARM, RAL, JV, RJS analyzed data; BPC, JLC, EBH, CMT, ARM, RAL, JV, RJS wrote the paper; BPC had primary responsibility of final content. All authors read and approved the final manuscript and authorship list.

STATEMENT OF INTERESTS

Declaration of personal interests: The remaining authors do not have personal interests to disclose. 
Bacteroides, Ruminococcaceae, Faecalibacterium prausnitzii) and 3 KEGG orthologs, of which two relate to carbohydrate metabolism.

Conclusions-In childhood IBS, a low FODMAP diet decreases abdominal pain frequency. Gut microbiome biomarkers may be associated with low FODMAP diet efficacy.

\section{Keywords}

Pediatric; Diet; IBS; Food

\section{INTRODUCTION}

Childhood irritable bowel syndrome (IBS) is a common functional gastrointestinal (GI) disorder affecting up to $20 \%$ of school-aged children and characterized by abdominal discomfort associated with defecation, or changes in stool frequency or stool form. ${ }^{1}$ Children with functional GI disorders frequently attribute some of their symptoms to specific foods, which negatively affect their quality of life. ${ }^{2}$

Fermentable carbohydrates such as lactose and fructose may be difficult to absorb and have been blamed for causing symptoms in children with chronic abdominal pain. ${ }^{3}$ The onset of these symptoms may be rapid, with an increase in GI symptoms in lactose intolerant adults with IBS occurring within three hours following a lactose challenge. ${ }^{4}$ The potential role of fermentable carbohydrates becomes more intriguing given their ever increasing consumption in the typical American childhood diet (TACD). ${ }^{5}$ However, controlled carbohydrate restriction dietary intervention trials in children with IBS have not demonstrated clear efficacy to date but primarily have focused on the restriction of only one (e.g., lactose) substrate at a time. ${ }^{6}$ Recently, the low FODMAP (fermentable oligosaccharides, disaccharides, monosaccharides, and polyols) diet, which comprehensively lowers the intake of several fermentable carbohydrates concurrently, has been shown to decrease GI symptoms in adults with IBS. ${ }^{7}$ The length of time needed for GI symptom amelioration on a low FODMAP diet in adult IBS is as little as 48 hours. ${ }^{8}$ A randomized controlled trial of a low FODMAP diet has not been conducted to date in children with IBS.

Despite the evidence supporting low FODMAP diet efficacy, $\geq 25 \%$ of adult IBS subjects do not improve on the diet. ${ }^{9}$ Although compliance may contribute to low FODMAP efficacy ${ }^{10}$, there is little information regarding other factors which may play a role. The hypothesized mechanism of action of a low FODMAP diet is related to decreased microbial fermentation of dietary carbohydrate leading to lower luminal osmolality and gas (e.g., hydrogen) generation. ${ }^{11}$ Several adult IBS studies have reported changes in specific stool bacterial composition while on the low FODMAP diet. ${ }^{12,13}$ However none of the studies have determined whether baseline gut microbiome composition may relate to low FODMAP diet efficacy or have completed comprehensive metagenomic evaluations of gut microbiome composition. Being able to predict who will respond to a low FODMAP diet may help avoid beginning this relatively challenging diet in those unlikely to respond. ${ }^{14}$

In a small, open label, pilot study, we previously demonstrated the potential benefit of a low FODMAP diet in children with IBS. ${ }^{15}$ Those who responded robustly to the diet were found 
to have a different microbiome composition as compared to those who did not. Given these results, in a separate group of children, we carried out a randomized, double blind, crossover trial to determine the effectiveness of a low FODMAP diet in childhood IBS. We hypothesized that a low FODMAP diet would decrease abdominal pain frequency and that children who responded to the diet would have a different microbiome composition and associated microbial metabolic capacity as compared to those who did not respond.

\section{METHODS}

Subjects

Children, ages 7 to 17 years, with Pediatric Rome III-defined IBS determined by completion of the pediatric Rome III GI symptom questionnaire were eligible. Potential subjects were identified by screening referrals to tertiary pediatric gastroenterology care for chronic abdominal pain and via community newsletters and internet advertisements. Parents and children were further screened by phone for inclusion and exclusion criteria and to establish current symptoms prior to enrollment as previously described. ${ }^{15}$ Study recruitment began September 2011 and ended December 2013. Baylor College of Medicine Institutional Review Board approval was obtained. The study was registered as ClinicalTrials.gov identifier NCT01339117.

\section{General Design}

Subjects completed a 7-day period in which they continued ingesting their habitual (baseline) diet (Figure 1). Following this baseline period, we employed a randomized, double-blind, crossover study design. Subjects received either a low FODMAP or typical American childhood diet (TACD) for 48 hours. After 48 hours on the first assigned diet, they returned to their habitual diet for 5 days. Following this 5-day washout period, they were crossed over to the other intervention diet for 48 hours. The randomization scheme was computer generated (www.randomization.com) with blocks of ten without stratification and with access to the scheme only provided to the United States Department of Agriculture (USDA) Children's Nutrition Research Center (CNRC) research dietitian.

\section{Stool Collection}

A stool sample was collected while the subjects were on their habitual diet (baseline period) as previously described. ${ }^{16}$ Briefly, a stool "hat" was placed on the toilet to capture the stool. The stool was immediately transferred to a sterile container and placed in the subjects' freezer. The stool then was transported on ice via courier to the investigators and stored at $-80^{\circ} \mathrm{C}$.

\section{Diets}

FODMAPs were defined as previously described. ${ }^{8}$ The low FODMAP diet contained 0.15 $\mathrm{g} \cdot \mathrm{kg}^{-1} \cdot \mathrm{day}^{-1}$ (maximum $9 \mathrm{~g} /$ day) of FODMAPs. The TACD contained $0.7 \mathrm{~g} \cdot \mathrm{kg}^{-1} \cdot \mathrm{day}^{-1}$ (maximum $50 \mathrm{~g} /$ day) of FODMAPs. Subjects were not informed that FODMAP content was being altered between the two provided diets. The meals were prepared at the USDA CNRC, and we attempted to match the two study diets with the baseline diet in terms of daily number of calories, protein, fat, and type of liquid/food consumed. Foods were selected from 
a general checklist of acceptable options that the child and a parent agreed could be consumed. When possible the same type of food or drink with different FODMAP content was provided during both dietary interventions. For example lactose-free vs. lactose containing milk or diet soda (with less fructose) vs. regular soda of the same brand were provided. At times, due to child preference, foods containing fats such as meats were increased to provide extra calories in cases where the FODMAP diet precluded adding more carbohydrate containing food. Children were provided a list of low FODMAP foods to consume if they remained hungry. The prepared foods were delivered to the subjects' residence for consumption. Following each diet, all food containers were returned to the CNRC and weighed to calculate the amount of food consumed during each dietary period. The Nutrition Data System for Research (University of Minnesota) version 2012 was used to analyze the food records.

\section{Measures}

The primary outcome measure (number of pain episodes) was captured through a Pain and Stool Diary over 24-hour periods for the seven days of the baseline period and for the two days of each dietary intervention. ${ }^{17}$ Abdominal pain location, severity, and duration were captured over three 8-hour time periods daily as previously described. ${ }^{17}$ Pain severity was measured on a $0-10$ Likert scale with 0 being "no pain at all" and 10 representing the "worst pain you can imagine." 17 Stools were characterized using the modified Bristol stool form chart for children. ${ }^{18}$ Children were subtyped based on stool form. ${ }^{1}$

Secondary outcome measures included associated daily GI symptoms (abdominal discomfort, bloating, flatus, nausea, heartburn) captured using a 0-10 Likert scale with 0 being "none" and 10 being "the worst." A composite GI symptom score was calculated by summing the associated daily GI symptoms in a manner consistent with other investigators evaluating dietary interventions. ${ }^{7}$

A food record was kept for three days during the baseline period and during each day of the intervention periods as previously described. ${ }^{15}$ The dietary components measured are shown in Table 1.

On the second day of each dietary intervention period, subjects collected hourly breath samples for hydrogen and methane. Samples were collected for $\geq 8$ hours, up to 15 hours. Breath samples were collected using a standard collection kit (Kidsampler System®, Quintron Instrument Co., Milwaukee, WI) and were analyzed for hydrogen, methane, and carbon dioxide (for normalization) as previously described (MicroLyzer® Model SC, QuintTron Instrument Company, Milwaukee, WI). ${ }^{15}$ Three children did not complete the breath testing and were not included in the gas production analyses.

\section{Microbiome Composition and Metabolic Capacity}

DNA extraction, bacterial 16S rRNA gene amplification, and 454 sequencing of 16S rRNA gene libraries were performed at the Texas Children's Microbiome Center as previously described. ${ }^{16}$ In brief, DNA was extracted from stool samples using a commercial DNA extraction kit (MO-BIO PowerSoil ${ }^{\circledR}$ DNA Isolation Kit, MO-BIO Laboratories) following the modified protocols described by the Human Microbiome Project. ${ }^{19}$ Individual sequence 
libraries were generated using bar-coded primers targeting the V3-V5 region of the $16 \mathrm{~S}$ rRNA gene. Following emulsion PCR, the PCR products were pooled and sequenced on the GS-FLX platform (454 Life Sciences/Roche).

The pooled sequence data were quality filtered, parsed by barcode, and screened for chimeras using the Quantitative Insights Into Microbial Ecology (QIIME) software package v 1.7.0. ${ }^{20}$ Sequences were screened for chimeras using the ChimeraSlayer algorithm ${ }^{21}$, and all potential chimeras were excluded from downstream analysis. Sequences with lengths shorter than $200 \mathrm{bp}$, average quality scores <20, ambiguous base calls, or mismatches to their barcode or sequencing primer also were excluded from downstream analysis. The quality filtered sequences were assigned to operational taxonomic units (OTUs; sequences that share $\geq 97 \%$ similarity) using a closed reference approach in QIIME with the UCLUST algorithm ${ }^{22}$ and Greengenes reference database (version 13_5). ${ }^{23}$ In cases where more than one OTU was assigned to the same organism at the species level, re-evaluation of all individual sequences belonging to those OTUs was performed using the UCLUST algorithm to confirm that the correct taxonomic attribution was made.

An average of 5570 high quality $16 \mathrm{~S}$ rRNA gene sequences were generated per stool sample (range: 2757-13194). Given the variation in library size and the potential for differences in sequencing depth to bias the calculation of diversity metrics, each library was randomly subsampled to contain 2700 sequences. All results presented here are based on these subsampled libraries. Alpha diversity metrics, including OTU richness, Shannon H, and the reciprocal Simpson index (1/D) were calculated using QIIME. Beta diversity metrics, including (UniFrac weighted or un-weighted), were calculated in QIIME.

Phylogenetic Investigation of Communities by Reconstruction of Unobserved States (PICRUSt) was used to generate functional metagenomic predictions by linking taxonomic information from the 16S rRNA gene sequences to KEGG (Kyoto Encyclopedia of Genes and Genomes) annotations of reference genomes. ${ }^{24,25}$ Orthologs of interest were evaluated by accessing their descriptive information at http://www.genome.jp/kegg/ko.html (date accessed: February 2, 2015).

Full sequence libraries were deposited in the National Center for Biotechnology Information Sequence Read Archive.

\section{Statistical Analyses}

The primary outcome measure was the number of daily abdominal pain episodes during each of the dietary intervention periods versus the baseline period. Abdominal pain scores are frequently used as primary outcome measures in childhood irritable bowel syndrome trials. ${ }^{26}$ Secondary outcome measures included median pain severity, the composite GI symptom score, and breath hydrogen and methane production during each of the dietary intervention periods versus the baseline period.

For gut microbiome biomarker analysis, Responders were classified as subjects who had a $250 \%$ decrease in the number of daily abdominal pain episodes during the low FODMAP dietary arm of the study and not to the TACD intervention. Non-Responders were classified 
as subjects who lacked $\geq 50 \%$ decrease in the frequency of abdominal pain episodes as compared to baseline during both the low FODMAP and TACD interventions. Subjects who had $250 \%$ decrease in frequency of abdominal pain episodes to both the low FODMAP and TACD interventions as well as those who had $250 \%$ decrease in frequency of abdominal pain episodes with the TACD intervention alone were classified as Placebo-responders. ${ }^{27,} 28$ A $\geq 50 \%$ decrease was chosen per recommendations for outcomes in IBS studies and in parallel with our previous work. ${ }^{15,29}$

Linear discriminant analysis effect size (LEfSe) (http://huttenhower.sph.harvard.edu/galaxy) was used to identify differences in taxa composition and KEGG orthologs between Responders and Non-Responders. ${ }^{30}$ LEfSe analysis includes a nonparametric factorial Kruskal-Wallis rank sum test to detect taxa with differential abundances. ${ }^{30}$ Linear discriminant analysis with bootstrapping more than 30 cycles was used to determine a linear discriminant analysis score to estimate the effect size between the two groups. ${ }^{30}$ Alpha values of 0.05 were used with a threshold on the logarithmic score of linear discriminant analysis being $\geq 2.0$ as published and used by others in the field. ${ }^{30,31}$

IBM Statistics (SPSS, version 22; Armonk, NY) was used for statistical evaluation. Comparisons between dietary intervention periods and between each dietary intervention period and baseline used paired Wilcoxon (continuous variables) and McNemar's testing for categorical variables. Only those completing both dietary interventions were included in the analysis. Data from the second day of each intervention was used as was convention at the time of the study. ${ }^{8}$ The number of abdominal pain episodes at baseline was normalized to a per day value to allow direct comparison to the test diets. Data are presented as mean \pm standard deviation for parametric data or median (25-75\% range) for non-parametric data unless otherwise specified. P-values $<0.05$ were considered statistically significant.

Based on previous work demonstrating that children with IBS had on average $0.5 \pm 0.4$ episodes of abdominal pain per day ${ }^{17}$ and identifying a $50 \%$ change in the frequency of abdominal pain as being clinically significant, we had estimated 33 children would be needed to complete the crossover trial with an $\alpha=0.05$ and power of $90 \%$ to detect a significant difference.

\section{RESULTS}

Fifty-two children were enrolled in this study, and 33 completed both arms of the crossover trial (Figure 1 ). The majority of dropouts (74\%) left the study prior to the start of any dietary intervention (i.e., during the baseline period). The remaining dropouts did not complete the Pain and Stool Diary (Figure 1). Of those completing the trial, 22 (67\%) were female and mean age was $11.5 \pm 3.0 \mathrm{yrs}$. There were $22(66.6 \%)$ Caucasians, 10 (30.3\%) Blacks, and 1 (3.0\%) Asian. Eleven (33\%) were of Hispanic ethnicity. IBS subtypes included: 24 (72.7\%) IBS-Constipation, 3 (9.1\%) IBS-Unsubtyped, 3 (9.1\%) IBS-Mixed, and 3 (9.1\%) IBSDiarrhea. 


\section{Comparison of Dietary Interventions (Table 1)}

Seventeen children began with the TACD, and 16 began with the low FODMAP diet. No adverse events were reported while on either diet. Compliance based on food records and weigh-ins between the TACD and low FODMAP diet was similar $(85.2 \% \pm 15 \%$ and $90.7 \%$ $\pm 10.8 \%$, respectively).

\section{Gastrointestinal Symptoms and Stooling}

Children had fewer daily abdominal pain episodes during the low FODMAP as compared to the TACD dietary intervention $(1.1 \pm 0.2$ (SEM) vs. $1.7 \pm 0.4$ pain episodes per day, respectively; $\mathrm{P}<0.05)$. Compared to baseline ( $1.4 \pm 0.2$ pain episodes per day), children had fewer daily abdominal pain episodes during the low FODMAP diet $(\mathrm{P}<0.01)$ but more episodes during the TACD dietary intervention $(\mathrm{P}<0.01)$. Median pain severity decreased on the low FODMAP $\operatorname{diet}[1(0-4), \mathrm{P}<0.001)$ and TACD [2.5 (0-4), $\mathrm{P}<0.01]$ as compared to baseline [4 (2.5-6)]. Eight subjects were categorized as Responders (had significant improvement on the low FODMAP diet only), 15 as Non-Responders (did not have significant improvement on the low FODMAP or TACD), and 10 as Placebo-responders (improved on both diets or only on the TACD diet).

The total composite GI score was lower on the low FODMAP diet versus TACD diet (5.8 \pm 6.2 versus $7.3 \pm 6.4$, respectively, $\mathrm{P}<0.05)$. However the composite GI symptom score was not significantly different from baseline $(6.2 \pm 6.0)$ during either the low FODMAP or TACD diet.

\section{Gas Production}

Breath hydrogen production was lower during the low FODMAP diet versus TACD diet (Figure 2a). Methane production did not differ between the two dietary periods (Figure 2b). Breath hydrogen production did not correlate with abdominal pain frequency during either dietary intervention period (data not shown).

Responders tended to have greater hydrogen production (ppm*hour) versus Non-responders during the TACD $(13,922 \pm 6170$ versus $9356 \pm 2681$, respectively; $\mathrm{P}=0.08)$. No differences in hydrogen production were noted between Responders versus Non-Responders during the low FODMAP Diet $(10,357 \pm 3158$ versus $8632 \pm 3493$, $\mathrm{P}=0.37)$. There were no significant differences with respect to methane production between the two groups (data not shown).

\section{Microbiome Signatures in Responders versus Non-responders}

LEfSe analysis identified 63 microbial OTUs enriched in Responders at baseline, of which 21 had a $\log { }^{10}$ LDA score greater than 2.5 (Table 2) and 4 OTUs enriched in NonResponders at baseline (Table 3). Responders to the low FODMAP diet were enriched at baseline in several OTUs with greater saccharolytic metabolic capacity within the family Bacteroidaceae (e.g., Bacteroides), order Clostridiales (e.g., Ruminococcaceae, Dorea, and Faecalibacterium prausnitzii) and family Erysipilotrichaceae (e.g., cc_115). The multiple OTUs assigned to F. prausnitzii in Responders were examined further and found to have a $0.003 \%$ erroneous sequence attribution, with all errors correct to the family level of 
Ruminococcaceae. Non-Responders were uniquely enriched at baseline in the genus Turicibacter from the family Turicibacteraceae.

LEfSe analysis identified 3 KEGG orthologs enriched at baseline in Responders (Table 4). Two of these three KEGG orthologs are related to FODMAP carbohydrate metabolism (LacI family transcriptional regulator and alpha- $\mathrm{N}$-arabinofuranosidase) with alpha-Narabinofuranosidase in particular being related to metabolism of carbohydrates found in wheat which are specifically avoided on the low FODMAP diet. ${ }^{7,} 32$ No KEGG orthologs were found to be enriched at baseline in Non-Responders.

There were no significant differences in microbial alpha diversity (Supplemental Table 1) or beta diversity between Responders and Non-Responders. No differences were found in baseline dietary composition between Responders and Non-Responders (data not shown).

\section{DISCUSSION}

Identifying effective therapies for children with IBS and determining which children are more likely to respond to a particular therapy is an important goal. To our knowledge, this study is the first controlled, double blind, randomized trial evaluating the efficacy of a low FODMAP diet in children with IBS. Equally important, we demonstrated that both baseline gut microbiome composition and microbial metabolic capacity are associated with FODMAP diet efficacy. If confirmed, these findings may pave the way toward personalized IBS dietary interventions and allow individuals to proceed with or avoid a dietary intervention in which adherence may be challenging. ${ }^{14}$

Subsequent to completing our study, Halmos et al. reported a double blind, randomized, crossover trial of an extended low FODMAP diet in adults with IBS. ${ }^{7}$ They showed an increasing ameliorating effect of a low FODMAP diet on pain symptoms beginning within the first day and increasing over the first seven days with a subsequent relative plateau over the next 14 days. ${ }^{7}$ Based on Halmos' data, a longer intervention timeframe in our study may have allowed for a greater effect size to emerge. Nonetheless, our findings of an amelioration in symptoms within 48 hours are further supported by a similarly designed randomized, crossover adult IBS trial. ${ }^{8}$ Furthermore, as in our study, Halmos et al. found an improvement in symptoms in those with IBS of different subtypes ${ }^{7}$, suggesting potential benefit irrespective of IBS subtype. Controlled studies evaluating the long term usage of a low FODMAP diet (i.e., several months or more) and subsequent effect on GI symptoms, gastrointestinal physiology, and microbiome composition and function within children and adults with IBS of various subtypes remains to be done to ensure long-term efficacy and safety. ${ }^{33}$

One of the presumed mediators of low FODMAP diet efficacy is the gut microbiome. ${ }^{9}$ It is believed that the low FODMAP diet results in decreased microbial fermentation and thus, less production of gas and osmotically active metabolites. ${ }^{11,34}$ Responders were enriched prior to the start of any dietary interventions in microbes from several taxa such as Bacteroides, Ruminococcaceae, and Dorea which have a large number of carbohydrateactive enzymes and as such may have greater saccharolytic potential. ${ }^{35,} 36$ Similarly, several 
OTUs assigned to Faecalibacterium prausnitzii also were found to be enriched at baseline in Responders. This species, which may be an indicator of intestinal health, ${ }^{37}$ is also able to ferment complex carbohydrates, such as inulin, which are avoided in the low FODMAP diet. ${ }^{38}$ Our findings suggest that identification of microbiota with greater saccharolytic capacity may serve as a biomarker for who may respond most robustly to FODMAP avoidance.

We found that members of the genus Turicibacter were uniquely enriched prior to the start of any dietary interventions in Non-Responders. Though these bacteria also have carbohydrate fermentative activity, particularly of grains fed to ruminant animals, ${ }^{39}$ their ability to ferment carbohydrates avoided in the low FODMAP diet may be limited. Turicibacter were recently found to decrease in abundance in mice fed fructooligosaccharides. ${ }^{40}$ In addition, a Turicibacter species was found to have specific carbohydrate requirements for growth including maltose and 5-ketogluconate. ${ }^{41}$ Our finding suggests Non-Responders to the diet may be enriched in bacteria prior to the start of the diet which are not well suited to ferment carbohydrates included in the FODMAP group. Future studies investigating gut microbiome composition more comprehensively, including nonbacterial microbiota such as fungi, and function at baseline and in response to a low FODMAP diet intervention in Responders vs. Non-Responders will help further elucidate the mechanism of the diet and may identify further therapeutic targets. ${ }^{42,} 43$

KEGG orthologs predicted to be enriched in those who became Responders included two related to carbohydrate metabolism. The majority of LacI transcription factors regulate carbohydrate utilization genes and allow for expression of these genes when the appropriate substrate or environmental condition is present. ${ }^{44,45}$ Alpha- $\mathrm{N}$-arabinofuranosidase acts on several substrates including arabinogalactans which may be found in foods such as wheat flour, an avoided food in the low FODMAP diet. ${ }^{32}$ The potential enrichment of these carbohydrate-related KEGG ortholog groups supports the premise that Responders likely have a gut microbiome with greater FODMAP saccharolytic potential.

As seen in an adult IBS trial, we found decreased hydrogen production during the low FODMAP diet as compared to the TACD. ${ }^{8}$ These findings suggest a difference in microbial fermentative metabolism that occurs as a function of diet. We found gas production did not correlate with pain frequency. Gas production may potentially be a fermentation marker rather than a direct inducer of symptoms. Other potential mechanisms for symptom generation include other microbial fermentation-derived metabolites (e.g., short chain fatty acids, bile acids) which may affect intestinal mucosal immune and barrier function. ${ }^{46}$ Host factors such as visceral hypersensitivity also may play a role. ${ }^{47}$ Adults with IBS-D with lactose intolerance were found to have increased rectal sensitivity, serum tumor necrosis factor-alpha, and gut mucosal inflammatory cells following lactose challenge versus healthy controls with lactose malabsorption. ${ }^{48}$ Whether these findings apply to children with IBS remains to be elucidated.

Subjects with IBS have a placebo response rate similar to that seen in organic GI and chronic pain disorders, and this may be a significant confounder of intervention efficacy. ${ }^{27}$ A crossover study design represents a traditional method by which the placebo response can 
be measured with subjects serving as their own control. ${ }^{49}$ Through this we determined who specifically responded to the low FODMAP diet and also were able to determine who specifically did not respond to any intervention. Children who responded to both dietary interventions or to the TACD were treated as Placebo-responders. ${ }^{27,28}$ Through this method we feel we were able to best approach the subsequent microbiome-related investigations consistent with recommendations by experts in the field. ${ }^{50}$

There are several strengths to our study. Strengths include a rigorous randomized, crossover, double blind design limiting bias. In addition, the study was prospective, using a validated pain and stool diary throughout. Another strength was provision of food to the subjects, resulting in good compliance. Study limitations included not having all enrollees complete the crossover study. We feel this may potentially be related to reluctance to carry out the protocol tasks given the vast majority dropped out before starting either of the dietary interventions thereby limiting the impact of dropouts on the results. Another limitation may be potential issues with the washout period in crossover studies. However, while dramatic dietary interventions have been shown to induce rapid changes in microbiome composition and metabolites, these measures return to baseline values within 5 days when returning to a habitual diet. ${ }^{51}$ In addition, we did not see a significant effect of diet order (i.e., low FODMAP or TACD first) in Responders. As such, the washout period used in our study is likely to have been adequate. Despite our best efforts to match the two diets in as many aspects as possible, another limitation may be differences in taste or other contents outside of FODMAP carbohydrates that may have affected outcomes. Future studies may consider providing identical foods but varying FODMAP content through liquids which are made to taste similarly. Finally, the study was conducted at a single center and future studies conducted at other institutions will lend greater generalizability to the results.

In conclusion, a low FODMAP diet appears to ameliorate GI symptoms in children with IBS. Those who responded robustly to the diet appear to have a different baseline microbiome composition, with greater saccharolytic capacity, than those who do not respond to a low FODMAP diet. Future research may determine whether evaluation of the gut microbiome may lead to personalized low FODMAP or other dietary intervention therapy in those with IBS.

\section{Supplementary Material}

Refer to Web version on PubMed Central for supplementary material.

\section{ACKNOWLEDGEMENTS}

We thank Jane Muir, Jacqueline Barrett, and Dr. Peter Gibson for their guidance with the dietary intervention; the CNRC kitchen team for food preparation; as well as Andrea Lopez, Mallory Balkin, Diana Lu, Linda Cao, Shannon Orlando, and Iqra Hussain for their research assistance.

Bruno Chumpitazi receives funding from the National Institutes of Health (NIH), QOL Medical Inc. and is a consultant for Mead Johnson Nutrition. Robert Shulman receives funding from the NIH, and Mead-Johnson Inc. and is a consultant for Nutrinia Inc. and Gerson Lehrman Group. James Versalovic receives funding from the NIH, and Biogaia $\mathrm{AB}$.

Declaration of funding interests: Funding was provided by the NASPGHAN Foundation/Nestle Nutrition Career Development Award and National Institutes of Health (NIH) K23 DK101688 (BPC). This study also was supported 
in part by NIH R01 NR05337 (RJS), NIH UH3 DK083990 (JV), the USDA/ARS under Cooperative Agreement No. 6250-51000-043 (RJS), and P30 DK56338 which funds the Texas Medical Center Digestive Disease Center.

\section{Abbreviations}

FODMAP Fermentable oligosaccharides disaccharides monosaccharides and polyols

IBS Irritable bowel syndrome

TACD Typical American childhood diet

\section{REFERENCES}

1. Self MM, Czyzewski DI, Chumpitazi BP, et al. Subtypes of irritable bowel syndrome in children and adolescents. Clin Gastroenterol Hepatol. 2014; 12(9):1468-73. [PubMed: 24486406]

2. Carlson MJ, Moore CE, Tsai CM, et al. Child and parent perceived food-induced gastrointestinal symptoms and quality of life in children with functional gastrointestinal disorders. J Acad Nutr Diet. 2014; 114(3):403-13. [PubMed: 24360501]

3. Barr RG, Levine MD, Watkins JB. Recurrent abdominal pain of childhood due to lactose intolerance. N Engl J Med. 1979; 300(26):1449-52. [PubMed: 449886]

4. Dainese R, Casellas F, Marine-Barjoan E, et al. Perception of lactose intolerance in irritable bowel syndrome patients. Eur J Gastroenterol Hepatol. 2014; 26(10):1167-75. [PubMed: 25089542]

5. Vos MB, Kimmons JE, Gillespie C, et al. Dietary fructose consumption among US children and adults: the Third National Health and Nutrition Examination Survey. Medscape J Med. 2008; 10(7): 160. [PubMed: 18769702]

6. Huertas-Ceballos AA, Logan S, Bennett C, et al. Dietary interventions for recurrent abdominal pain (RAP) and irritable bowel syndrome (IBS) in childhood. Cochrane Database Syst Rev. 2009; 1:CD003019. [PubMed: 19160214]

7. Halmos EP, Power VA, Shepherd SJ, et al. A Diet Low in FODMAPs Reduces Symptoms of Irritable Bowel Syndrome. Gastroenterology. 2014; 146(1):67-75. [PubMed: 24076059]

8. Ong DK, Mitchell SB, Barrett JS, et al. Manipulation of dietary short chain carbohydrates alters the pattern of gas production and genesis of symptoms in irritable bowel syndrome. J Gastroenterol Hepatol. 2010; 25(8):1366-73. [PubMed: 20659225]

9. Gibson PR, Shepherd SJ. Food choice as a key management strategy for functional gastrointestinal symptoms. Am J Gastroenterol. 2012; 107(5):657-66. [PubMed: 22488077]

10. Shepherd SJ, Gibson PR. Fructose malabsorption and symptoms of irritable bowel syndrome: guidelines for effective dietary management. J Am Diet Assoc. 2006; 106(10):1631-9. Epub 2006/09/27. [PubMed: 17000196]

11. Murray K, Wilkinson-Smith V, Hoad C, et al. Differential effects of FODMAPs (fermentable oligo-, di-, mono-saccharides and polyols) on small and large intestinal contents in healthy subjects shown by MRI. Am J Gastroenterol. 2014; 109(1):110-9. [PubMed: 24247211]

12. Halmos EP, Christophersen CT, Bird AR, et al. Diets that differ in their FODMAP content alter the colonic luminal microenvironment. Gut. 2015; 64(1):93-100. [PubMed: 25016597]

13. Staudacher HM, Lomer MC, Anderson JL, et al. Fermentable carbohydrate restriction reduces luminal bifidobacteria and gastrointestinal symptoms in patients with irritable bowel syndrome. $\mathrm{J}$ Nutr. 2012; 142(8):1510-8. [PubMed: 22739368]

14. de Roest RH, Dobbs BR, Chapman BA, et al. The low FODMAP diet improves gastrointestinal symptoms in patients with irritable bowel syndrome: a prospective study. Int J Clin Pract. 2013; 67(9):895-903. [PubMed: 23701141]

15. Chumpitazi BP, Hollister EB, Oezguen N, et al. Gut microbiota influences low fermentable substrate diet efficacy in children with irritable bowel syndrome. Gut Microbes. 2014; 5(2):16575. [PubMed: 24637601] 
16. Saulnier DM, Riehle K, Mistretta TA, et al. Gastrointestinal Microbiome Signatures of Pediatric Patients With Irritable Bowel Syndrome. Gastroenterology. 2011; 141(5):1782-91. [PubMed: 21741921]

17. Shulman RJ, Eakin MN, Jarrett M, et al. Characteristics of pain and stooling in children with recurrent abdominal pain. J Pediatr Gastroenterol Nutr. 2007; 44(2):203-8. [PubMed: 17255832]

18. Chumpitazi BP, Lane MM, Czyzewski DI, et al. Creation and initial evaluation of a Stool Form Scale for children. J Pediatr. 2010; 157(4):594-7. [PubMed: 20826285]

19. Human Microbiome Project C. Structure, function and diversity of the healthy human microbiome. Nature. 2012; 486(7402):207-14. [PubMed: 22699609]

20. Caporaso JG, Kuczynski J, Stombaugh J, et al. QIIME allows analysis of high-throughput community sequencing data. Nat Methods. 2010; 7(5):335-6. [PubMed: 20383131]

21. Haas BJ, Gevers D, Earl AM, et al. Chimeric 16S rRNA sequence formation and detection in Sanger and 454-pyrosequenced PCR amplicons. Genome Res. 2011; 21(3):494-504. [PubMed: 21212162]

22. Edgar RC. Search and clustering orders of magnitude faster than BLAST. Bioinformatics. 2010; 26(19):2460-1. [PubMed: 20709691]

23. DeSantis TZ, Hugenholtz P, Larsen N, et al. Greengenes, a chimera-checked 16S rRNA gene database and workbench compatible with ARB. Appl Environ Microbiol. 2006; 72(7):5069-72. [PubMed: 16820507]

24. Langille MG, Zaneveld J, Caporaso JG, et al. Predictive functional profiling of microbial communities using 16S rRNA marker gene sequences. Nature Biotechnol. 2013; 31(9):814-21. [PubMed: 23975157]

25. Kanehisa M, Goto S, Sato Y, et al. KEGG for integration and interpretation of large-scale molecular data sets. Nucleic Acids Res. 2012; 40(Database issue):D109-14. [PubMed: 22080510]

26. Vlieger AM, Menko-Frankenhuis C, Wolfkamp SC, et al. Hypnotherapy for children with functional abdominal pain or irritable bowel syndrome: a randomized controlled trial. Gastroenterology. 2007; 133(5):1430-6. [PubMed: 17919634]

27. Enck P, Horing B, Weimer K, et al. Placebo responses and placebo effects in functional bowel disorders. Eur J Gastroenterol Hepatol. 2012; 24(1):1-8. [PubMed: 21915059]

28. Enck P, Klosterhalfen S, Weimer K, et al. The placebo response in clinical trials: more questions than answers. Philos Trans R Soc Lond B, Biol Sci. 2011; 366(1572):1889-95. [PubMed: 21576146]

29. Irvine EJ, Whitehead WE, Chey WD, et al. Design of treatment trials for functional gastrointestinal disorders. Gastroenterology. 2006; 130(5):1538-51. [PubMed: 16678567]

30. Segata N, Izard J, Waldron L, et al. Metagenomic biomarker discovery and explanation. Genome Biol. 2011; 12(6):R60. [PubMed: 21702898]

31. Ma J, Prince AL, Bader D, et al. High-fat maternal diet during pregnancy persistently alters the offspring microbiome in a primate model. Nature Commun. 2014; 5:3889. [PubMed: 24846660]

32. Saeed F, Pasha I, Anjum FM, et al. Arabinoxylans and arabinogalactans: a comprehensive treatise. Crit Rev Food Sci Nutr. 2011; 51(5):467-76. [PubMed: 21491271]

33. Rao SS, Yu S, Fedewa A. Systematic review: dietary fibre and FODMAP-restricted diet in the management of constipation and irritable bowel syndrome. Aliment Pharmacol Ther. 2015; 41(12):1256-70. [PubMed: 25903636]

34. Lomer MC. Review article: the aetiology, diagnosis, mechanisms and clinical evidence for food intolerance. Aliment Pharmacol Ther. 2015; 41(3):262-75. [PubMed: 25471897]

35. Flint HJ, Scott KP, Duncan SH, et al. Microbial degradation of complex carbohydrates in the gut. Gut Microbes. 2012; 3(4):289-306. [PubMed: 22572875]

36. Taras D, Simmering R, Collins MD, et al. Reclassification of Eubacterium formicigenerans Holdeman and Moore 1974 as Dorea formicigenerans gen. nov., comb. nov., and description of Dorea longicatena sp. nov., isolated from human faeces. Int J Syst Evol Microbiol. 2002; 52:4238. Pt 2. [PubMed: 11931151]

37. Varela E, Manichanh C, Gallart M, et al. Colonisation by Faecalibacterium prausnitzii and maintenance of clinical remission in patients with ulcerative colitis. Aliment Pharmacol Ther. 2013; 38(2):151-61. [PubMed: 23725320] 
38. Miquel S, Martin R, Rossi O, et al. Faecalibacterium prausnitzii and human intestinal health. Curr Opin Microbiol. 2013; 16(3):255-61. [PubMed: 23831042]

39. Mao S, Zhang R, Wang D, et al. The diversity of the fecal bacterial community and its relationship with the concentration of volatile fatty acids in the feces during subacute rumen acidosis in dairy cows. BMC Vet Res. 2012; 8:237. [PubMed: 23217205]

40. Everard A, Lazarevic V, Gaia N, et al. Microbiome of prebiotic-treated mice reveals novel targets involved in host response during obesity. ISME J. 2014; 8(10):2116-30. [PubMed: 24694712]

41. Bosshard PP, Zbinden R, Altwegg M. Turicibacter sanguinis gen. nov., sp. nov., a novel anaerobic, Gram-positive bacterium. Int J Syst Evol Microbiol. 2002; 52:1263-6. Pt 4. [PubMed: 12148638]

42. Dupont HL. Review article: evidence for the role of gut microbiota in irritable bowel syndrome and its potential influence on therapeutic targets. Aliment Pharmacol Ther. 2014; 39(10):1033-42. [PubMed: 24665829]

43. Wang ZK, Yang YS, Stefka AT, et al. Review article: fungal microbiota and digestive diseases. Aliment Pharmacol Ther. 2014; 39(8):751-66. [PubMed: 24612332]

44. Ravcheev DA, Khoroshkin MS, Laikova ON, et al. Comparative genomics and evolution of regulons of the LacI-family transcription factors. Front Microbiol. 2014; 5:294. [PubMed: 24966856]

45. O'Connell KJ, Motherway MO, Liedtke A, et al. Transcription of two adjacent carbohydrate utilization gene clusters in Bifidobacterium breve UCC2003 is controlled by LacI- and repressor open reading frame kinase (ROK)-type regulators. Appl Environ Microbiol. 2014; 80(12):360414. [PubMed: 24705323]

46. Rajilic-Stojanovic M, Jonkers DM, Salonen A, et al. Intestinal Microbiota And Diet in IBS: Causes, Consequences, or Epiphenomena? Am J Gastroenterol. 2015; 110(2):278-87. [PubMed: 25623659]

47. Shepherd SJ, Lomer MC, Gibson PR. Short-chain carbohydrates and functional gastrointestinal disorders. Am J Gastroenterol. 2013; 108(5):707-17. [PubMed: 23588241]

48. Yang J, Fox M, Cong Y, et al. Lactose intolerance in irritable bowel syndrome patients with diarrhoea: the roles of anxiety, activation of the innate mucosal immune system and visceral sensitivity. Aliment Pharmacol Ther. 2014; 39(3):302-11. [PubMed: 24308871]

49. Enck P, Klosterhalfen S, Zipfel S. Novel study designs to investigate the placebo response. BMC Med Res Methodol. 2011; 11:90. [PubMed: 21663609]

50. Goodrich JK, Di Rienzi SC, Poole AC, et al. Conducting a microbiome study. Cell. 2014; 158(2): 250-62. [PubMed: 25036628]

51. David LA, Maurice CF, Carmody RN, et al. Diet rapidly and reproducibly alters the human gut microbiome. Nature. 2014; 505(7484):559-63. [PubMed: 24336217] 


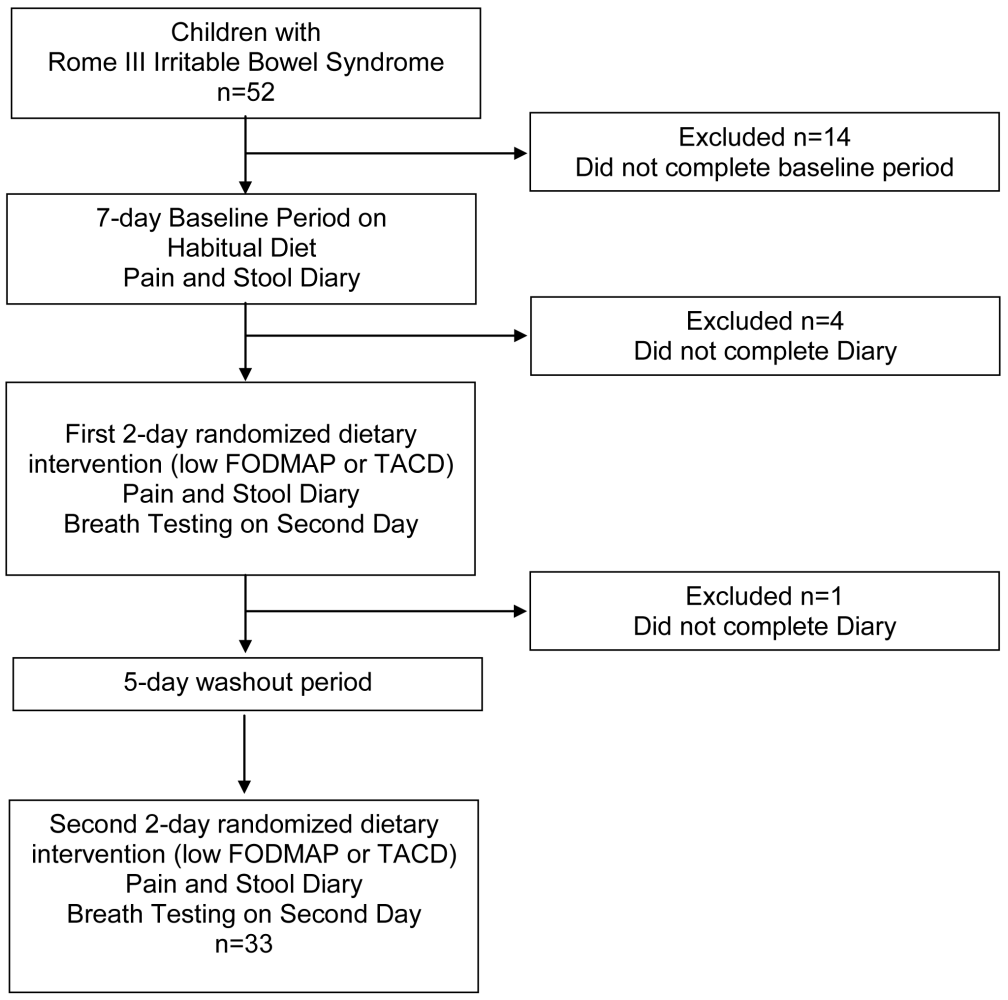

Figure 1.

Trial flow sheet. FODMAP = fermentable oligosaccharides, disaccharides, monosaccharides, and polyols. TACD = typical American childhood diet. 

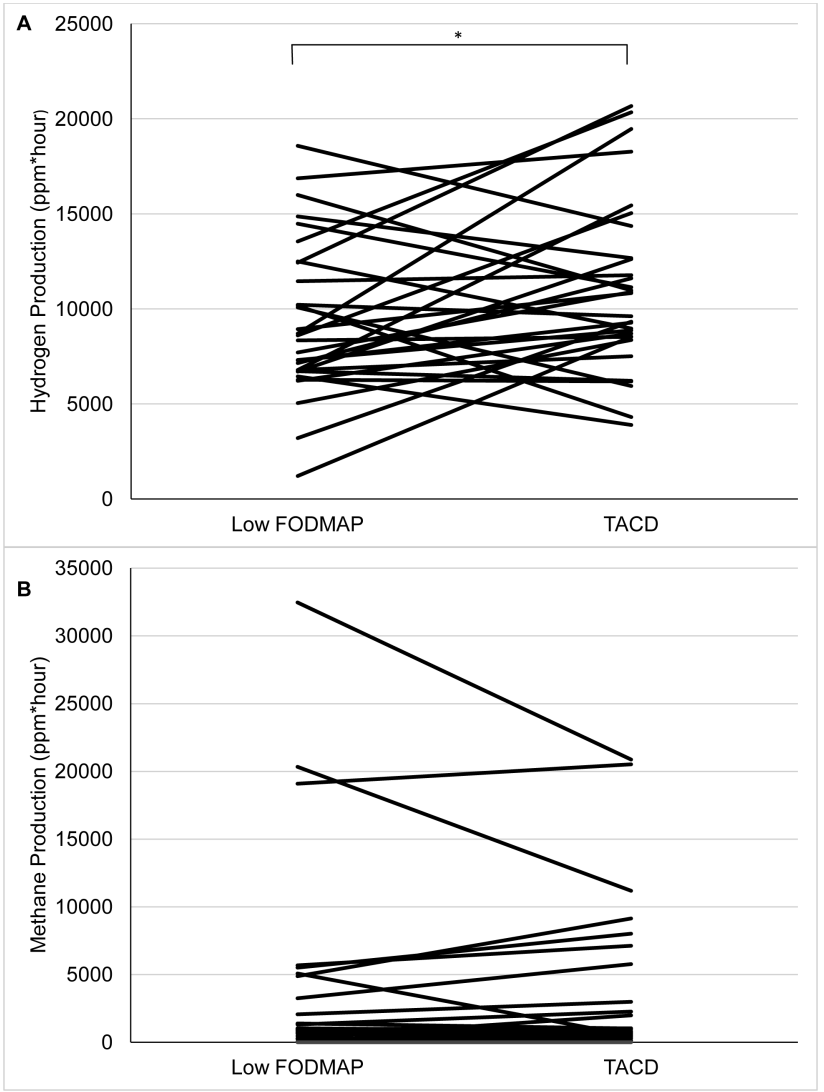

Figure 2.

A) Comparison of hydrogen and B) methane gas production during the second day of the low fermentable oligosaccharides, disaccharides, monosaccharides, and polyols (FODMAP) diet versus a typical American childhood diet (TACD). 


\section{Table 1}

Nutritional Composition by Component of the Provided Low FODMAP and Typical American Childhood Diet in a Randomized Crossover Study in Children with IBS

\begin{tabular}{|l|c|c|}
\hline \multicolumn{1}{|c|}{ Component } & $\begin{array}{c}\text { Low FODMAP } \\
\text { Diet }\end{array}$ & $\begin{array}{c}\text { Typical American } \\
\text { Childhood Diet }\end{array}$ \\
\hline Energy, kcal & $1801 \pm 69$ & $1800 \pm 56$ \\
\hline Protein, g & $76.5 \pm 3.9$ & $70.2 \pm 2.6$ \\
\hline Fat, g & $63.5 \pm 2.9^{\#}$ & $52.1 \pm 2.1$ \\
\hline Carbohydrate, g & $239.5 \pm 9.4^{\#}$ & $268.8 \pm 10.9$ \\
\hline Dietary Fiber, g & $20.2 \pm 1.1$ & $16.8 \pm 0.7$ \\
\hline Glucose, g & $23.4 \pm 1.6^{*}$ & $34.2 \pm 2.2$ \\
\hline Fructose, g & $21.1 \pm 1.6^{*}$ & $57.9 \pm 4.1$ \\
\hline Lactose, g & $1.3 \pm 1.0^{*}$ & $4.1 \pm 0.6$ \\
\hline Sucrose, g & $31.1 \pm 2.0$ & $34.3 \pm 1.7$ \\
\hline Maltose, g & $3.2 \pm 0.2$ & $3.5 \pm 0.2$ \\
\hline Polyols, g & $0.8 \pm 0.6^{*}$ & $4.4 \pm 0.3$ \\
\hline
\end{tabular}

Mean \pm SEM

FODMAP $=$ fermentable oligosaccharides disaccharides monosaccharides and polyols Paired t-test analysis

${ }^{\#}=\mathrm{P}<0.05$ comparison to typical American diet

* $=\mathrm{P}<0.001$ comparison to typical American diet 


\section{Table 2}

Operational Taxonomic Units (OTUs) with Assigned Taxonomy by Lowest Taxonomic Rank Enriched at Baseline in Responders ( $n=8)$ versus Non-Responders $(n=15)$ to a Low FODMAP diet per Linear Discriminant Analysis (LDA) Effect Size Analysis. OTUs with LDA scores (log 10) greater than 2.5 are included. OTUs are best matches and not all OTUs could be identified to the species level.

\begin{tabular}{|l|l|l|l|}
\hline \multicolumn{1}{|c|}{ OTU } & \multicolumn{1}{|c|}{ Taxa } & LDA Score & P-value \\
\hline 2921213 & Bacteroides (genus) & 4.11 & .0054 \\
\hline 358781 & Ruminococcaceae (family) & 3.98 & .0048 \\
\hline 175441 & Faecalibacterium prausnitzii (species) & 3.53 & .032 \\
\hline 178081 & Ruminococcaceae (family) & 3.49 & .025 \\
\hline 4446898 & Bacteroides (genus) & 3.48 & .042 \\
\hline 297057 & Bacteroides (genus) & 3.22 & .013 \\
\hline 4417335 & Bacteroides (genus) & 3.14 & .03 \\
\hline 187505 & Dorea (genus) & 3.1 & .048 \\
\hline 171559 & Bacteroides (genus) & 3.06 & .015 \\
\hline 4463532 & Clostridiales (order) & 2.86 & .023 \\
\hline 175175 & Lachnospiraceae (family) & 2.78 & .047 \\
\hline 3991008 & Faecalibacterium prausnitzii (species) & 2.77 & .025 \\
\hline 4143073 & Clostridiales (order) & 2.7 & .032 \\
\hline 4342682 & Faecalibacterium prausnitzii (species) & 2.66 & .046 \\
\hline 180341 & Dorea (genus) & 2.64 & .048 \\
\hline 183267 & Lachnospiraceae (family) & 2.62 & .047 \\
\hline 565289 & CC_115 (genus) & 2.62 & .048 \\
\hline 177878 & Faecalibacterium prausnitzii (species) & 2.6 & .038 \\
\hline 136526 & Erysipelotrichaceae (family) & 2.57 & .078 \\
\hline 341724 & Ruminococcaceae (family) & 2.53 & .0065 \\
\hline 157631 & Ruminococcaceae (family) & 2.52 & .048 \\
\hline
\end{tabular}


Table 3

Operational Taxonomic Unit (OTU) with Assigned Taxonomy by Lowest Taxonomic Rank Enriched at Baseline in Non-Responders $(n=15)$ versus Responders $(n=8)$ to a Low FODMAP diet per Linear Discriminant Analysis (LDA) Effect Size Analysis. OTUs are best matches and not all OTUs could be identified to the species level.

\begin{tabular}{|l|l|l|l|}
\hline \multicolumn{1}{|c|}{ OTU } & \multicolumn{1}{c|}{ Taxa } & LDA Score & P-value \\
\hline 4358723 & Bacteroides (genus) & 2.8 & .033 \\
\hline 347529 & Turicibacter (genus) & 2.65 & .026 \\
\hline 17859 & Clostridiales (order) & 2.6 & .044 \\
\hline 248902 & Turicibacter (genus) & 2.59 & .015 \\
\hline
\end{tabular}


Table 4

Kyoto Encyclopedia Genes and Genomes (KEGG) Orthologs as Identified by Phylogenetic Investigation of Communities by Reconstruction of Unobserved States (PICRUSt) Enriched at Baseline in Responders $(n=8)$ versus Non-Responders $(\mathrm{n}=15)$ to a Low FODMAP Diet per Linear Discriminant Analysis (LDA) Effect Size Analysis.

\begin{tabular}{|l|l|l|l|}
\hline KEGG Ortholog & \multicolumn{1}{|c|}{ Description } & LDA Score & P-value \\
\hline K02529 & LacI family transcriptional regulator & 2.23 & .028 \\
\hline K01209 & $\begin{array}{l}\text { Alpha-N-arabinofuranosidase } \\
{[\text { EC.3.2.1.55] }}\end{array}$ & 2.11 & .045 \\
\hline K03496 & Chromosome partitioning protein & 2.03 & .024 \\
\hline
\end{tabular}

